University of Nebraska - Lincoln

DigitalCommons@University of Nebraska - Lincoln

3-18-1996

\title{
Laser Injection of Ultrashort Electron Pulses into Wakefield Plasma Waves
}

Donald P. Umstadter

University of Nebraska-Lincoln, donald.umstadter@unl.edu

J.K. Kim

University of Michigan, Ann Arbor

E. Dodd

University of Michigan, Ann Arbor

Follow this and additional works at: https://digitalcommons.unl.edu/physicsumstadter

Part of the Physics Commons

Umstadter, Donald P.; Kim, J.K.; and Dodd, E., "Laser Injection of Ultrashort Electron Pulses into Wakefield Plasma Waves" (1996). Donald Umstadter Publications. 31.

https://digitalcommons.unl.edu/physicsumstadter/31

This Article is brought to you for free and open access by the Research Papers in Physics and Astronomy at DigitalCommons@University of Nebraska - Lincoln. It has been accepted for inclusion in Donald Umstadter Publications by an authorized administrator of DigitalCommons@University of Nebraska - Lincoln. 


\title{
Laser Injection of Ultrashort Electron Pulses into Wakefield Plasma Waves
}

\author{
D. Umstadter, J. K. Kim, and E. Dodd \\ Center for Ultrafast Optical Science, University of Michigan, Ann Arbor, Michigan 48109
}

(Received 11 December 1995)

\begin{abstract}
A novel laser-plasma-based source of relativistic electrons is described. It involves a combination of orthogonally directed laser beams, which are focused in a plasma. One beam excites a wakefield electron plasma wave. Another locally alters the trajectory of some of the electrons in such a way that they can be accelerated and trapped by the wave. With currently available table-top terawatt lasers, a single ultrashort-duration electron bunch can be accelerated to multi-MeV energies in a fraction of a millimeter, with femtosecond synchronization between the light pulse, the electron bunch, and the plasma wave. Both analytical and numerical-simulation results are presented.
\end{abstract}

PACS numbers: 52.75.Di, 29.25.Bx, 41.75.Lx, 52.40.Nk

The recent development of compact high-peak-power lasers [1] has spurred renewed interest [2-4] in electron acceleration by the use of the ultrahigh-electric-field gradients $(>10 \mathrm{GeV} / \mathrm{m})$ of laser-driven plasma waves. Because they are not limited by dielectric breakdown, their electric field gradients are 3 orders of magnitude higher than those in conventional RF linacs. The physical mechanism underlying them is simple. When an intense laser pulse propagates through a plasma, its so-called ponderomotive force displaces plasma electrons. Since this force is proportional to the laser intensity gradient, it will be directed primarily in the longitudinal direction if the laser pulse length is much shorter than its focal spotsize and primarily in the transverse direction in the opposite case. Because the ions remain stationary, due to their much larger inertia, a residual charge imbalance results after the laser pulse passes by. Thus a large amplitude plasma wave will be "resonantly" driven in the wake of the laser pulse if its pulse width approximately equals a plasma-wave period. The resulting electrostatic laser wakefield propagates in the same direction and at nearly the same phase velocity as the group velocity of the laser pulse.

Normally, electrons oscillating in the plasma wave under the wave-breaking limit cannot be accelerated by the wakefield since they are out of phase with it. Electrons that are not part of the plasma wave, however, can become trapped-i.e., continuously accelerated-by the plasma wave, provided that they are moving in the correct phase at nearly the phase velocity of the wave [5]. Since this velocity is close to the speed of light, it is generally thought that the required preacceleration can be accomplished only with a conventional linac. In this paper, we show instead that it can simply be done with an additional laser pulse. The basic idea is that once a laser wakefield is excited by the longitudinal ponderomotive force of one laser pulse (the pump pulse), the transverse ponderomotive force of a second, orthogonally directed laser pulse (the injection pulse) can then be used to locally alter the trajectories of some of the plasma wave electrons such that they become in phase with the wave's electric field and thus accelerated to the trapping velocity [6].
By permitting the use of laser wakefields in the injection stage, this concept not only dispenses with the need to merge two dissimilar technologies, as in proposed hybrid systems [4], it also significantly increases the capabilities and applications of plasma-based accelerators. It is easier to synchronize in phase and overlap in space the processes of electron injection and acceleration by employing the same basic mechanism for both. Improved electron beam emittance may result from the increased field gradient in the first acceleration stage, by minimizing the time during which electrons are nonrelativistic and thus most susceptible to space charge effects. A device based on this concept can be used either as a stand-alone accelerator system or as an injector for either conventional or plasma-based high-energy accelerators. Both the energetic electrons and the high-energy photons into which they can be converted have numerous industrial, medical, and scientific applications. For instance, this technique produces a single ultrashort-duration (fs) electron or x-ray pulse (without the need for pulse selection or beam compression), which is synchronized with an ultrashort laser pulse, and thus suitable for the study of ultrafast dynamics [7]. This is 2 orders of magnitude shorter duration for a single pulse of electrons than ever produced by a conventional linac [8].

We begin by calculating the trapping threshold. Although multiple pulses are more efficient [9], for the sake of simplicity we will first consider single-pump-pulse wakefield excitation [2,3], assuming pump and wake to be copropagating along the $z$ axis. The pump laser pulse is described by $\mathbf{a}=e \mathbf{A}_{\perp} / m_{e} c^{2}$, where $\mathbf{A}_{\perp}$ is the transverse vector potential of the pump. Denoting $\Phi$ and $v_{g}$ as the electrostatic potential and the group velocity of the wake respectively, the $1 \mathrm{D}$ governing equation for the wakefield plasma wave, characterized by a plasma frequency $\omega_{p}$, is given by [2]

$$
\frac{d^{2} \phi}{d \zeta^{2}}=k_{p}^{2} \gamma_{g}^{2}\left[\beta_{g}\left(1-\frac{1+a^{2}}{\gamma_{g}^{2}\left(1+\phi^{2}\right)}\right)^{-1 / 2}-1\right],
$$

where $\phi=e \Phi / m_{e} c^{2}, \beta_{g}=v_{g} / c \approx \beta_{\phi}, \gamma_{g}=(1-$ $\left.\beta_{g}^{2}\right)^{-1 / 2} \approx \gamma_{\phi}$, and the plasma wave number $k_{p}=w_{p} / c$. 
The electric field of the wake experienced by background electrons and their momentum in the plasma wave are $\pi / 2$ out of phase with each other and thus almost no electrons can be trapped. However, if the amplitude of the electric field of the plasma wave is very large, a small amount of dephasing of the electron momentum with respect to the field can result in the trapping and acceleration of these electrons.

Thus the next step is to send an injection pulse along the $y$ axis into the plasma channel. Assuming the injection pulse has a Gaussian profile both temporally and spatially, and is described by the pulse width $\tau$, and the beam radius at the focal point $r_{0}$, as well as amplitude $b_{0}$, then the normalized vector potential for the injection pulse $\mathbf{b}$ can be written as $\mathbf{b}=\mathbf{b}_{\text {env }}(z, t) e^{i(\omega t-k y)}$, where $\mathbf{b}_{\text {env }}(z, t)=\mathbf{b}_{0} \exp \left[-\left(z / r_{0}\right)^{2}\right] \exp \left\{-4\left[\left(t-t_{c}\right) / \tau\right]^{2}\right\}, t_{c}$ is the time the peak of the injection pulse crosses the $z$ axis, and the $\omega$ is the laser frequency. The focal point of the injection pulse is at $z=0$ and $y=0$. The ponderomotive force of the injection pulse in the transverse direction [10], $F_{\text {pond }}=\left[\left(m_{e} c^{2} / r_{0}^{2}\right) z /\left(1+b_{0}^{2} / 2\right)^{2}\right] b_{\mathrm{env}}^{2}(z, t)$, which is in both positive and negative $z$ directions, produces bidirectional drift motion of the electrons in the plasma wave. Those electrons that obtain a ponderomotive drift velocity in the same direction as the pump pulse are velocity dephased with respect to, and injected into, the wakefield. If this velocity exceeds a certain threshold velocity, then the electron will be trapped in the wakefield.

In order to get the approximate functional form for the change of the dimensionless relativistic momentum $\Delta(\gamma \beta)$ of the electron, denoting $\tau_{p}$ as a plasma period, $\tau / \tau_{p} \ll 1$ is assumed, which asserts that the injection pulse is essentially a $\delta$ function in time. Using an electron whose plasma oscillation center is $z=r_{0} / 2$, the ponderomotive force assumes positive maximum value. The injection pulse produces

$$
\Delta(\gamma \beta)=\Delta p_{0} \delta\left(t-t_{c}\right),
$$

where $\quad \Delta p_{0}=\sqrt{\pi / 2} \exp (-1 / 2) b_{0}^{2} \tau / 4\left(1+b_{0}^{2} / 2\right)^{1 / 2} r_{0}$. Equation (2) describes the injection process as instantaneous at $t=t_{c}$ allowing electrons to make a transition across the separatrix. This assumption can be justified if $r_{0}$ of the injection pulse is on the order of $\lambda_{p}$, much larger than excursion distance of the electron, $z_{0} \approx\left(\lambda_{p} / 2 \pi\right) \phi$. The argument of the $\delta$ function determines $\omega_{p} t_{c}$, the phase of plasma wave when the electron has the sudden jump of its momentum. Because of the $\delta$ function in Eq. (2), the momentum of the electron experiences discontinuity at $t=t_{c}$ and is dephased with respect to other background plasma wave electrons. Aliasing $\gamma \beta$ with $p$, we have a new value for the momentum of the electron $p^{\prime}=p\left(t_{c}\right)+\Delta p_{0}$. We link this momentum with the injection energy required for trapping [11], $\Gamma=\gamma_{\phi}^{2}\left\{\epsilon+1 / \gamma_{\phi}-\beta_{\phi}\left|\left(\epsilon+2 / \gamma_{\phi}\right) \epsilon\right|^{1 / 2}\right\}, \quad$ where $\epsilon=\phi_{\max }-\phi\left(t_{c}\right)$.
Equating $p^{\prime}$ and $\left(\Gamma^{2}-1\right)^{1 / 2}$, we have the threshold amplitude for the injection pulse

$$
b_{\text {th }}=\frac{S}{2}\left[1+\left(1+16 / S^{2}\right)^{1 / 2}\right]^{1 / 2},
$$

where $S=\sqrt{8 / \pi} \exp (1 / 2)\left\{\left(\Gamma^{2}-1\right)^{1 / 2}+\Delta p_{0}\right\}$, which is the dashed line plotted in Fig. 1.

To test the concept in greater detail, by using background instead of test electrons and including the effect of the plasma-wave electric field, the relativistic equation of motion, with the Lorentz force, is solved numerically in two dimensions,

$$
\frac{d}{d t}\left(\gamma m_{e} \mathbf{v}\right)=q \mathbf{E}_{\mathrm{epw}}+\mathbf{F}_{\text {pond }},
$$

where $\gamma=\left[1-(v / c)^{2}\right]^{-1 / 2}$ and $\mathbf{E}_{\text {epw }}$ is the electric field of the plasma wave in $z$ direction, and the electron moves in the $y-z$ plane.

We make the following assumptions: The group velocity of the wake $v_{g}$ is approximately equal to $c$ and is a constant (the quasistatic approximation); there is linear polarization for the injection pulse along the $z$ axis; no wake is produced by the injection pulse; and the plasma wave channel is one dimensional. A value of $a_{0}=1$, corresponding to $\phi=0.7$, was used. By varying the values of both $t_{c}$ and $b_{0}$, it is found, as shown in the inset of Fig. 1, that $t_{c}$ significantly affects the threshold amplitude for the injection pulse $b_{\text {th }}$ since it provides synchronization between the acceleration phase of the wake and the ponderomotive push by the injection pulse. The solid line in Fig. 1 represents the results of a set of simulations obtained by varying $a_{0}$ but with $t_{c}$ held constant at its optimal value $t_{c}=1.95 \tau_{p}$ (see Fig. 1) which corresponds approximately to the maximum of the potential overlapping the peak of the injection pulse as it crosses the $z$ axis. Since the injection pulse not only has a transverse ponderomotive force but a longitudinal force as well, it can increase an

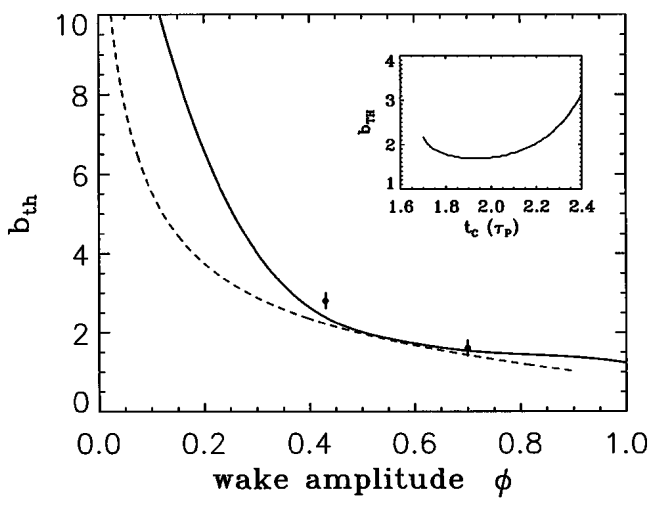

FIG. 1. The trapping threshold $b_{\text {th }}$ plotted vs the plasma-wave amplitude $\phi$. The dashed line represents the results of Eq. (3) and the solid line, Eq. (4). The trapping region is above the curves. The results of PIC simulations are shown by the two points; the error bars are from finite temperature effects. Inset: $b_{\text {th }}$ vs $t_{c}$, valid only along $y=0$. 
electron's momentum in the $y$ direction, orthogonal to the desired direction, that of plasma-wave propagation. However, the relativistic momentum conservation law asserts that $\beta_{z}$ and $\beta_{y}$ must satisfy $\beta_{y} /\left(1-\beta_{z}^{2}-\beta_{y}^{2}\right)^{1 / 2}=C$, where the constant $C$ is a measure of the effect of the longitudinal ponderomotive force, which is 0.374 in our case. This constant of motion prevents electrons from drifting in the $y$ direction out of a 1D plasma channel $\left(r_{\mathrm{epw}} \gg \lambda_{p}\right.$ where $r_{\mathrm{epw}}$ is the waist size of the channel). Once injected, $\beta_{y}$ decreases as the electron is accelerated. For instance, the transverse displacement after $t=40 \tau_{p}$ was $10 \mu \mathrm{m}$ or a distance of $\lambda_{p}$, and after $t=80 \tau_{p}$ it was only $1.3 \lambda_{p}$.

In order to fully test the principle of optical injection, including the effect of the injection pulse's wake, a particle-in-cell simulation (PIC) was performed. This particular code [9] has one spatial and three velocity dimensions and is fully relativistic. The pump pulse has wavelength $1 \mu \mathrm{m}$, and pulse length $10 \mu \mathrm{m}$, or $33 \mathrm{fs}$. The injection pulse is linearly polarized such that its magnetic field is set to be parallel to that of the pump pulse. The injection pulse has a spatial profile given by a Gaussian shape with a spot radius of $5 \mu \mathrm{m}$. The spatial domain size for the simulation is $10 \lambda_{p}$.

A simulation was run with $a_{0}=1(\phi=0.7)$ in order to compare with the results from Eq. (4). The position and the intensity of the injection pulse were varied in order to find the optimal trapping threshold intensity. A value of $b_{\mathrm{th}}=1.6$ was found by increasing $b_{0}$ until we obtain one or more trapped electrons. Figure 2 shows the velocity of one of these trapped electrons as a function of time, along with the phase space trajectory plotted in the inset of Fig. 2. As can be seen in Fig. 2, the gradual velocity dephasing along the $z$ direction by the injection pulse gave the electron enough boost for trapping. This value of $b_{\text {th }}$ and another obtained at a lower value of $\phi\left(a_{0}\right)$ are both
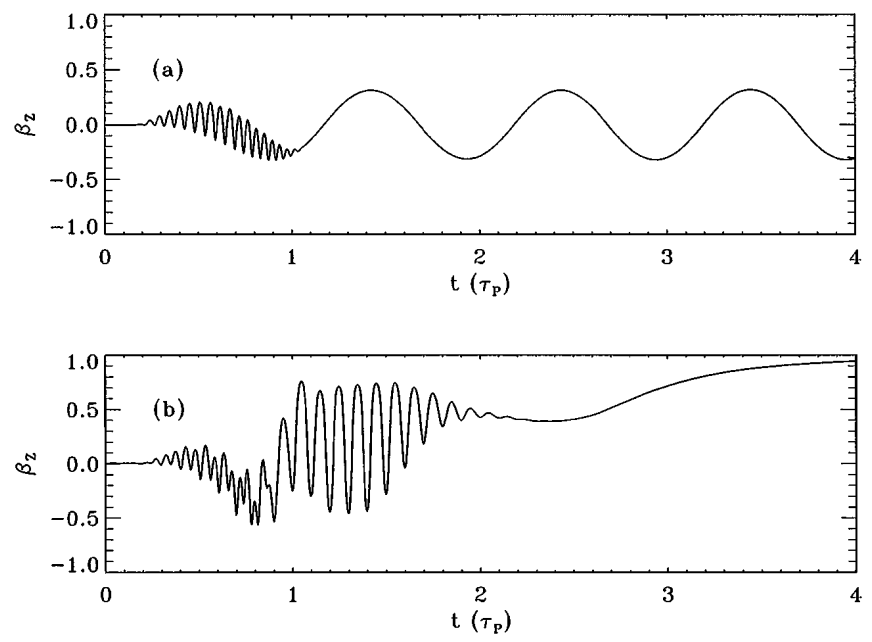

FIG. 2. (a) The velocity of an electron in the plasma wave without injection pulse, and (b) the velocity of an electron that becomes trapped, plotted as a function of time (in units of a plasma period $\tau_{p}$ ). plotted as points in Fig. 1. Since they agree with the values predicted by a 2D solution of Eq. (4) (the solid line), it appears that the assumption of only one spatial dimension in the PIC code has a negligible effect on $b_{\text {th }}$.

Figure 3 shows the output from a simulation with $b_{0}=2$; shown are the pump intensity a, the wakefield, and the longitudinal momentum $(\gamma \beta)_{z}$ of a number of the simulation electrons. The field of the pump and the wake are normalized to the quiver velocity. The pulse width of the injection pulse is equal to $\tau_{p}$. The normalized momentum $(\gamma \beta)_{z}$ distinguishes the electron beam from fast background electrons. Electrons that are not trapped by the second bucket (circled), 70 fs behind the pump pulse, fall into the next buckets. The plasma wave appears to be damped by beam loading. In an actual 2D case, more buckets could be filled as the injection pulse traverses the plasma wave. However, with tight focusing for the injection pulse, injection should only occur over a depth of focus centered on the $z$ axis, which in this case is approximately equal to a laser spotsize or a plasma wavelength. Thus the 1D simulation is a good representation of the $2 \mathrm{D}$ case.

Since the first two injected electron bunches have different average energies, it is possible to use a magnetic field to pick out only the first one. It has an average energy of $13.6 \mathrm{MeV}$, with an energy spread of $2.3 \mathrm{MeV}$, or $17.3 \%$. Its pulse length is $10 \mathrm{fs}$, or $3 \mu \mathrm{m}$. During the simulation the electrons travel approximately $160 \mu \mathrm{m}$, implying an average field gradient of $0.85 \mathrm{GeV} / \mathrm{cm}$, which is $85 \%$ of the maximum field gradient $1 \mathrm{GeV} / \mathrm{cm}$. Of course, a longer acceleration distance results in a narrower energy spread, both in absolute terms (from bunching) and relative terms, from higher final energies. For

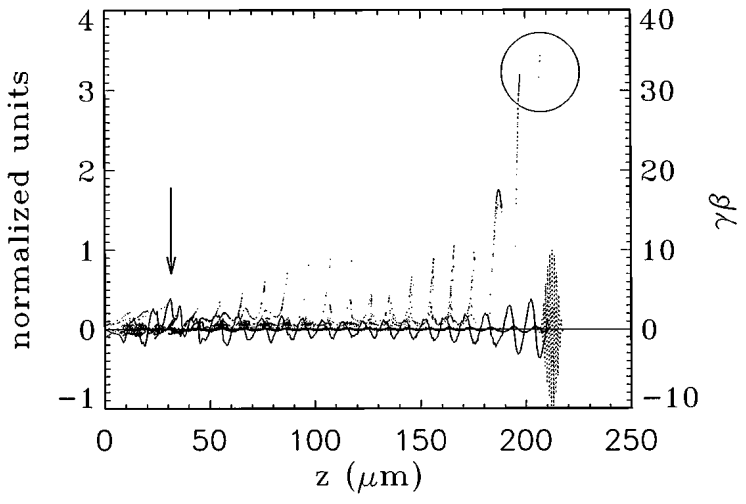

FIG. 3. A PIC simulation with an injection pulse of $b_{0}=$ 2.0; shown are the pump intensity $\mathbf{a}$, the wakefield, and the longitudinal momentum $(\gamma \beta)_{z}$ of a number of the simulation electrons. By generating a series of "fresh" acceleration buckets, the pump laser pulse, propagating from left to right, is leading the trapped electrons out of the region disturbed by the injection pulse at $z=30 \mu \mathrm{m}$, indicated by the arrow. The main part of a group of electrons that were trapped in the simulation is circled. 
instance, after $250 \mu \mathrm{m}$, the average energy is $21.2 \mathrm{MeV}$, with an energy spread of $1.3 \mathrm{MeV}$, or $6 \%$.

The actual number of trapped electrons in 3D can be estimated based on the number trapped in the 1D simulation and assuming a trapping volume determined in the following manner. Based on Eq. (4), we estimate a trapping length along $z$ at $y=0$ approximately given by $l_{z} \sim 4.0 \mu \mathrm{m}$. In the PIC code it was found to be $l_{z} \sim 2.0 \mu \mathrm{m}$. We can account for this narrowing by the observed spatial-profile modification of the plasma wave by the injection pulse. (Only $8 \%$ of the electrons in this region were actually trapped in the simulation.) Because of the change in phase $\left(t_{c}\right)$ that occurs as the laser pulse and the plasma wave propagate in orthogonal directions, $l_{z}$ will be reduced off axis, eventually vanishing at $y=$ $\pm 1.7 \mu \mathrm{m} \mu \mathrm{m}$. Both $l_{z}$ and $l_{y}$ can be increased either by increasing $b_{0}$ well beyond $b_{\text {th }}$ or by use of longer injection pulses, which increases the time over which the ponderomotive force acts.

It only remains to determine the trapping length in the direction perpendicular to the plane of incidence $l_{x}$. We assume loose focusing of the pump pulse and a circular focal spot size diameter of $50 \mu \mathrm{m}$ (corresponding to a pump power of 13.4 TW). Cylindrical focusing could be used for the injection pulse such that it could be focused tightly to a width of $10 \mu \mathrm{m}$ along $z$, but loosely to a length of $50 \mu \mathrm{m}$ along $x$, to match the diameter of the pump (corresponding to an injection power of $11 \mathrm{TW}$ ). (Also, by producing a uniform intensity gradient in the $z$ direction, cylindrical focusing gives those electrons located along the entire $x$ axis, not just at $x=0$, a boost only in the direction parallel to the $z$ axis.) Thus, using $l_{x}=50 \mu \mathrm{m}$, we estimate a value of greater than $10^{8}$ for the number of trapped electrons. This number can be increased by injecting electrons that are derived not just from the background, as discussed above, but also from the process of photoionization. Instead of using a fully ionized

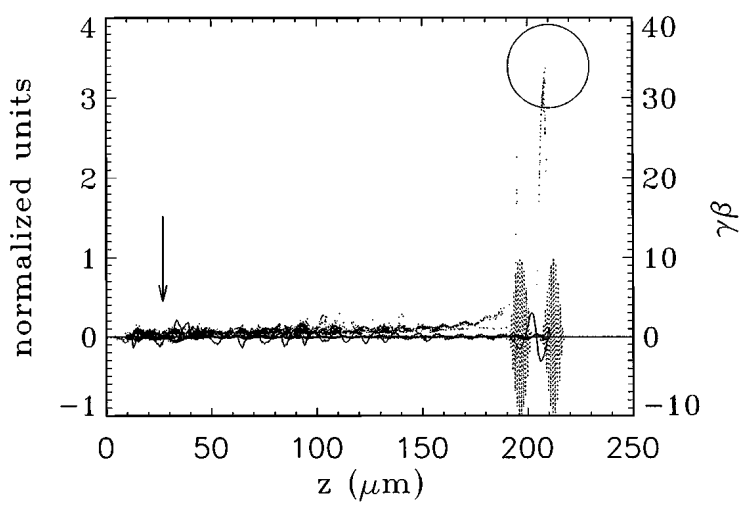

FIG. 4. A PIC simulation with a second antiresonant pump pulse to create only a single acceleration bucket, and thus a single electron bunch. Same parameters as in Fig. 3 except $\tau=3 \lambda_{p}$ for the injection pulse. The arrow pointing $z=30$ indicates where the injection pulse passed through. plasma (as above), one would use a medium with deeply bound inner shell electrons, which have an appearance intensity for tunneling ionization [12] below that of the injection pulse but above that of the pump pulse, a topic of a future publication.

In another simulation, as shown in Figure 4, by using a second antiresonant pump pulse $[9,13]$ to create only a single acceleration bucket, we have eliminated the electrons that get trapped in the later buckets. This also permitted the use of a longer duration $\left(\tau=3 \lambda_{p}\right)$ injection pulse, reducing its longitudinal ponderomotive force and thus the electron energy spread, but without filling more than one bucket. The same result might be achieved with a single pump pulse by using beam loading to damp the wave.

In this paper, using both analysis and PIC-code simulations, we have demonstrated a new concept for linear acceleration of electrons, in which a laser optically injects electrons into a wakefield plasma wave. One conclusion is that the threshold intensity of the injection pulse for trapping decreases with increasing $\phi$ until the point where $\phi \approx 0.6$ (corresponding to where the injection beam has a comparable intensity to the pump beam $b_{0}=a_{0} \sim 1$ ), after which $b_{\text {th }}$ becomes independent of $\phi$.

We would like to acknowledge the support of the NSF and to thank Torsten Neubert and Gerard Mourou for many valuable discussions.

[1] P. Maine et al., IEEE J. Quantum Electron. 24, 398 (1988); G. Mourou and D. Umstadter, Phys. Fluids B 4, 2315 (1992); M.D. Perry and G. Mourou, Science 264, 917 (1994).

[2] P. Sprangle et al., Appl. Phys. Lett. 53, 2146 (1988).

[3] T. Tajima and J. M. Dawson, Phys. Rev. Lett. 43, 267 (1979); L. M. Gorbunov and V. I. Kirsanov, Sov. Phys. JETP 66, 290 (1987); H. Hamster et al., Phys. Rev. Lett. 71, 2725 (1993); K. Nakajima et al., ibid. 74, 4428 (1995).

[4] See, e.g., Advanced Accelerator Concepts, Fontana, WI, 1994, edited by P. Schoessow, AIP Conf. Proc. No. 335 (AIP Press, New York, 1995), and references cited therein.

[5] E. Esarey and M. Pilloff, Phys. Plasmas 2, 1432 (1995).

[6] E. Dodd, J. K. Kim, and D. Umstadter, in Proceedings of the 37th Annual Meeting of the American Physical Society, Division of Plasma Physics, Louisville, KY, 1995.

[7] Ultrafast Phenomena IX, edited by P.F. Barbara, W. H. Knox, G. A. Mourou, and A. H. Zewail (Springer-Verlag, Berlin, 1994), and references cited therein.

[8] M. Uesaka et al., Phys. Rev. E 50, 3068 (1994).

[9] D. Umstadter, E. Esarey, and J. Kim, Phys. Rev. Lett. 72, 1224 (1994); D. Umstadter et al., Phys. Rev. E 51, 3484 (1995).

[10] E. L. Lindman and M. A. Stroscio, Nucl. Fusion 3, 619 (1977).

[11] T. Katsouleas et al., in Laser Accereration of Particles, edited by C. Joshi and T. C. Katsouleas, AIP Conf. Proc. No. 130 (AIP, New York, 1985).

[12] L. V. Keldysh, Sov. Phys. JETP 20, 1307 (1965).

[13] S. C. Wilks et al., Phys. Rev. Lett. 62, 2600 (1989). 